\title{
NONLINEAR CONTROL OF TEMPERATURE PROFILE OF UNSTABLE HEAT CONDUCTION SYSTEMS: A PORT HAMILTONIAN APPROACH
}

\author{
HOANG NGOC HA ${ }^{1}$, PHAN DINH TUAN ${ }^{2}$ \\ ${ }^{1}$ Dept. of Control and Chemical Process Engineering, University of Technology, VNU-HCM; \\ Email: ha.hoang@hcmut.edu.vn \\ ${ }^{2}$ Hochiminh City University of Natural Resources and Environment;
}

\begin{abstract}
choss
Abstract. This paper focuses on boundary control of distributed parameter systems (also called infinite dimensional systems). More precisely, a passivity based approach for the stabilization of temperature profile inside a well-insulated bar with heat conduction in a one-dimensional system described by parabolic partial differential equations (PDEs) is developed. This approach is motivated by an appropriate model reduction schema using the finite difference approximation method. On this basis, it allows to discretize and then, write the original parabolic PDEs into a Port Hamiltonian $(\mathrm{PH})$ representation. From this, the boundary control input is therefore synthesized using passive tools to stabilize the temperature at a desired reference profile asymptotically. In particular, a simple proportional passive controller with a relaxing condition for the control gain matrix is adopted. The infinite dimensional nature of the original distributed parameter system in the $\mathrm{PH}$ framework is also discussed. Numerical simulations illustrate the application of the developments.
\end{abstract}

Keywords. Port Hamiltonian framework, passivity, boundary control, model reduction.

\section{INTRODUCTION}

In this paper, the authors deal with open systems in which (unstable) heat conduction processes take place. In general, such processes belong to irreversible thermodynamic systems and are distributed in space and time. As a matter of fact, their dynamics are described by parabolic partial differential equations (PDEs) [1-3].

The distributed parameter process systems are usually highly nonlinear due to constitutive equations (for example chemical reaction kinetics, transport equations such as Fick's law or Fourier's law, etc.). Recent developments and theoretical challenges for controlling such systems can be found in $[4,5]$, and references therein. The main issues to be considered further can be summarized as follows:

- How to stabilize an unstable solution (if there exists) of the PDEs?

- How to explore and show the stabilization properties as well as the performances (response, robustness...) of the controlled dynamics?

It can be shown that the control synthesis and design for the distributed parameter systems have been broadly studied in the literature [6-9]. On the one hand, a very natural approach for control synthesis and design is to spatially discretize by approximating equations or solutions of the original PDEs using finite difference method, finite volume or Galerkin's methods [10-12]. The

(c) 2015 Vietnam Academy of Science \& Technology 
goal is to obtain a set of ordinary differential equations (ODEs) for which the nonlinear control strategies specially developed for the finite dimensional systems [13-15] can be applied. Let us cite for example, [16] for predictive control of transport reaction processes, [17-19], with robust control of parabolic PDE systems using classical Lyapunov based approach and [20] for passivity based control of a reduced port controlled Hamiltonian model for the shallow water equations. On the other hand, spectral methods (such as proper orthogonal decomposition [21] or Hammerstein modeling approach [22], symmetry groups and invariance conditions [23,24], geometric pseudo-spectral method [11] and energy based discretization [12] provide powerful tools to handle the dynamics described by PDEs directly. All these allow reducing the dimensionality of the system before synthesizing the feedback controllers. However, these approaches involve heavily mathematical calculations and do not exhibit any links to physico-chemical properties of the system under consideration. On the contrary, irreversible thermodynamics based stabilization has been recently developed for transport reaction systems $[7-9,25]$. The results proposed in $[7-9,25]$ are quite interesting and open research perspectives from both theoretical and practical viewpoints.

This paper focuses on the stabilization of the temperature profile of unstable heat conduction processes in the Port Hamiltonian ( $\mathrm{PH}$ ) framework ${ }^{1}$. To achieve this goal, a model reduction schema using the finite difference method is applied to write the original parabolic PDEs into the PH representation. From this, a proportional feedback controller is synthesized using passive properties to stabilize the system dynamics asymptotically. Contrary to the previous works, the contributions of this work are to show that a relaxing condition for the gain matrix $K$ of the proposed proportional controller (i.e., $K=K^{T} \geq 0$ instead of $K=K^{T}>0$ ) can also be used for the stabilization.

This paper is organized as follows. The PH framework based control is briefly reminded in Section 2. The (one-dimensional) unstable heat conduction process inside a homogeneous metal bar is presented in Section 3. A model reduction schema using the finite difference method for passivity based control is then proposed. The distributed parameter nature of the system in the infinite dimensional $\mathrm{PH}$ framework is also discussed in this section. Section 4 is dedicated to numerical simulations to illustrate the developments and show the effectiveness of the proposed approach. Section 5 ends the paper with concluding remarks and perspectives.

Notations: The following notations are considered throughout this paper:

- Let $\Re$ denote the set of all real numbers.

- $m, n$ are positive integers.

- Tcan either be the temperature or be used for the matrix transpose.

\section{HAMILTONIAN FORMALISM BASED CONTROL}

Port controlled Hamiltonian systems with dissipation are given by [26, 27]:

$$
\left\{\begin{array}{l}
\frac{d x}{d t}=[J(x)-R(x)] \frac{\partial H(x)}{\partial x}+g(x) u \\
y=g(x)^{T} \frac{\partial H(x)}{\partial x}
\end{array}\right.
$$

\footnotetext{
${ }^{1}$ We refer the reader to [26-28] for more details on the mathematical descriptions and control of portcontrolled Hamiltonian systems. Contrary to electromechanical systems where the link between the dissipation and energy is well established in the PH framework, the extension of $\mathrm{PH}$ framework to (bio) chemical processes usable both for the stability analysis and control design remains open [29].
} 
where:

$x=x(t) \in \Re^{n}$ is the state vector;

$u, y \in \Re^{m} \quad(m \leq n)$ are the control input and its conjugated power port variable respectively; this means that the unit of the scalar product $u^{T} y$ is power;

The smooth function $H(x): \Re^{n} \rightarrow \Re$ represents the Hamiltonian storage function ${ }^{2}$;

The interconnection matrix $J(x)=-J(x)^{T}$ and the damping matrix $R(x)=R(x)^{T} \geq 0$ are called structure matrices. $J(x)$ corresponds to reversible energy transfer between the different physical domains of the system, e.g. material one or thermal one. $R(x)$ represents the irreversible energy transfer between the different physical domains of the system;

$g(x)$ is the $n \times m$ input-state map.

The energy balance immediately follows from (1):

$$
\frac{d H(x)}{d t}=-\left[\frac{\partial H(x)}{\partial x}\right]^{T} R(x)\left[\frac{\partial H(x)}{\partial x}\right]+u^{T} y .
$$

The system (1) is passive in the sense that the dissipation given by,

$$
d=-\left[\frac{\partial H(x)}{\partial x}\right]^{T} R(x)\left[\frac{\partial H(x)}{\partial x}\right]
$$

is negative semi-definite and the Hamiltonian storage function $H(x)$ is bounded from below $[14,15]$. The amount of $d$ defined by (3) characterizes the irreversibility (for example energy lost due to friction/damping in mechanical systems or due to resistance in $R L C$ electrical system [26,27] or due to entropy production in the CSTR networks [29]). From (2) and (3), if the system (1) is passive then the following passivity inequality holds:

$$
\frac{d H(x)}{d t} \leq u^{T} y
$$

A methodology for controlling the Hamiltonian models described by (1) using Interconnection and Damping Assignment Passivity-Based Control (IDA-PBC) approach is given in [28].

\section{THE 1-D UNSTABLE HEAT CONDUCTION SYSTEM}

\subsection{Mathematical model}

Let us consider a one-dimensional unstable heat conduction system as sketched in Fig. 1.

$$
T(z, t)
$$

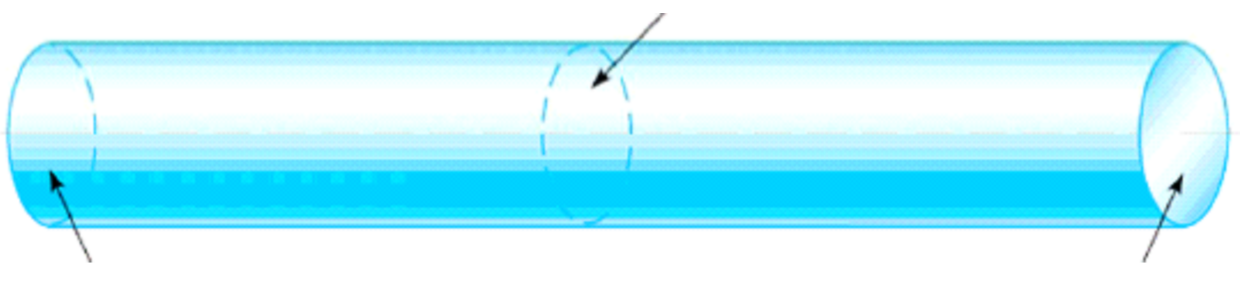

$$
z=0
$$

$$
z=L
$$

\footnotetext{
${ }^{2}$ The gradient of the Hamiltonian storage function with respect to $\mathrm{x}$ is denoted by $\frac{\partial H(x)}{\partial x}$.
} 
The heat conduction is assumed to be in the axial direction zonly. The volume expansivity is negligible. The evolution of the temperature within the bar is then established using balance energy and it is governed by the following parabolic partial differential equation (PDE) [1-3,30]:

$$
\rho c \frac{\partial T(z, t)}{\partial t}=\lambda \frac{\partial^{2} T(z, t)}{\partial z^{2}}
$$

(5) can be rewritten in an equivalent form:

$$
\frac{\partial T(z, t)}{\partial t}=D \frac{\partial^{2} T(z, t)}{\partial z^{2}}
$$

where $D=\frac{\lambda}{\rho c}$.

In Eq. (5), let us note that spatial variable $z \in\left[\begin{array}{ll}0 & L\end{array}\right]$ and time $t \in\left[\begin{array}{ll}0 & +\infty\end{array}\right)$. The parameter $\rho\left(\mathrm{g} / \mathrm{cm}^{3}\right)$ is the mass density, $c(\mathrm{~J} /(\mathrm{gK}))$ is the specific heat capacity and $\lambda(W /(\mathrm{cm} \mathrm{K}))$ is the heat conduction coefficient.

In addition, we assume that the evolution of the temperature governed by (6) is subject to the Dirichlet boundary condition and the initial condition as follows:

$$
\left\{\begin{array}{c}
T(z=0, t)=T_{l} \\
T(z=L, t)=T_{r}
\end{array}\right.
$$

and,

$$
T(z, t=0)=T^{\text {init }}(z)
$$

MAIN OBJECTIVE: The goal of this work is twofold. First, it shows that the system dynamics (6) in its deviation form can be written into the $\mathrm{PH}$ representation with a quadratic Hamiltonian storage function by considering an appropriate spatial discretization schema. Second, thanks to natural dissipation resulting from the proposed model reduction schema, a (simple) proportional feedback controller can be derived for the purpose of the stabilization of the temperature $T(z, t)$ at the desired reference profile $T^{*}(z)$ where the boundary variables $\left[\begin{array}{lll}T_{l}(t) & T_{r}(t)\end{array}\right]$ are used as the manipulated variables.

\subsection{Model reduction schema}

In this subsection, it is shown that the dynamics (6) written into its deviation form is a $\mathrm{PH}$ system using the finite difference approximation method. In what follows, the notation $T^{*}(z)$ refers to the stationary state ${ }^{3}$ of the dynamics (6) subject to the conditions (7)(8). It is worth noting that such a stationary state $T^{*}(z)$ fulfills the following equation:

$$
\frac{\partial T^{*}(z)}{\partial t}=D \frac{\partial^{2} T^{*}(z)}{\partial z^{2}} \equiv 0
$$

Let us denote the deviation variable by $\bar{T}(z, t)=T(z, t)-T^{*}(z)$. From this, subtracting (9) from (6) yields:

\footnotetext{
${ }^{3}$ All time derivatives vanish at this state or the time becomes very large, e.g., goes to infinity.
} 


$$
\frac{\partial \bar{T}(z, t)}{\partial t}=D \frac{\partial^{2} \bar{T}(z, t)}{\partial z^{2}} .
$$

As a consequence, (10) is also a parabolic PDE and subject to the boundary and initial conditions as follows:

$$
\left\{\begin{array}{l}
\bar{T}(z=0, t)=T_{l}(t)-T^{*}(0) \equiv \bar{T}_{l}(t) \\
\bar{T}(z=L, t)=T_{r}(t)-T^{*}(L) \equiv \bar{T}_{r}(t)
\end{array}\right.
$$

and,

$$
\bar{T}(z, t=0)=T^{i n i t}(z)-T^{*}(z) .
$$

The approach used to approximate the solution to (10) involves the finite difference method [10]. First let us select an integer $N>0$ and define the step size $h=\frac{L}{N}$. The grid points for this situation are $z_{i}$, where $z_{i}=i h$, for $i=0 \ldots N$. Let us denote the value of the deviation variable calculated at the grid point $z_{i}, \quad i=0 \ldots N$ by $\bar{T}\left(z_{i}, t\right)=\bar{T}_{i}(t)$.

The following proposition shows that the system dynamics (10) can be written into the PH representation (1) using the model reduction based on the finite difference method [10].

\section{Proposition 1. Model reduction using the finite difference method}

The system dynamics given by (10) in its reduced form using the finite difference method is a PH system (1) with state variables $x=\left(\begin{array}{l}\bar{T}_{1}(t) \\ \bar{T}_{2}(t) \\ \vdots \\ \bar{T}_{(N-2)}(t) \\ \bar{T}_{(N-1)}(t)\end{array}\right) \in \Re^{(N-1)}$, control input $u=\left(\begin{array}{l}\bar{T}_{l}(t) \\ 0 \\ \vdots \\ 0 \\ \bar{T}_{r}(t)\end{array}\right) \in$

$\Re^{(N-1)}$, output $y=\frac{D}{h^{2}}\left(\begin{array}{l}\bar{T}_{1}(t) \\ \bar{T}_{2}(t) \\ \vdots \\ \bar{T}_{(N-2)}(t) \\ \bar{T}_{(N-1)}(t)\end{array}\right) \in \Re^{(N-1)}, g(x)=\frac{D}{h^{2}} \in \Re$, structure matrices $J(x)=$ $0 \in \Re^{(N-1) \times(N-1)}$ and $R(x)=\frac{D}{h^{2}}\left(\begin{array}{ccccc}2 & -1 & 0 & \ldots & 0 \\ -1 & 2 & -1 & \ldots & 0 \\ 0 & -1 & 2 & \ldots & 0 \\ \vdots & \vdots & \vdots & \ldots & -1 \\ 0 & 0 & 0 & -1 & 2\end{array}\right) \in \Re^{(N-1) \times(N-1)}$. Furthermore, the Hamiltonian storage function is given by $H(x)=\frac{1}{2} x^{T} x \geq 0$.

\section{Proof.}

Using the finite difference method [10], the central difference approximation of the second order derivative is given as follows:

$$
\left.\frac{\partial^{2} \bar{T}(z, t)}{\partial z^{2}}\right|_{z=z_{i}} \approx \frac{\bar{T}_{i+1}(t)-2 \bar{T}_{i}(i)+\bar{T}_{i-1}(t)}{h^{2}}, i=1 \ldots(N-1) .
$$


Note also that $\bar{T}_{0}(t) \equiv \bar{T}_{l}(t)$ and $\bar{T}_{N}(t) \equiv \bar{T}_{r}(t)$. Next, we discretize (10) using (13) for the grid points $z_{i}, \quad i=1 \ldots(N-1)$. From this, it leads to:

$$
\begin{aligned}
& \frac{d}{d t} \underbrace{\left(\begin{array}{l}
\bar{T}_{1}(t) \\
\bar{T}_{2}(t) \\
\bar{T}_{3}(t) \\
\vdots \\
\bar{T}_{(N-1)}(t)
\end{array}\right)}_{x}=\underbrace{\frac{D}{h^{2}}\left(\begin{array}{ccccc}
-2 & 1 & 0 & \ldots & 0 \\
1 & -2 & 1 & \ldots & 0 \\
0 & 1 & -2 & \ldots & 0 \\
\vdots & \ldots & \ldots & \ldots & 1 \\
0 & 0 & 0 & 1 & -2
\end{array}\right)}_{-R(x)} \underbrace{\left(\begin{array}{l}
\bar{T}_{1}(t) \\
\bar{T}_{2}(t) \\
\bar{T}_{3}(t) \\
\vdots \\
\bar{T}_{(N-1)}(t)
\end{array}\right)}_{\frac{\partial H(x)}{\partial x}}+\underbrace{\frac{D}{h^{2}}}_{g(x)} u \\
& \text { with } u=\left(\begin{array}{l}
u_{1} \\
0 \\
\vdots \\
0 \\
u_{N-1}
\end{array}\right)=\left(\begin{array}{l}
\bar{T}_{0}(t) \\
0 \\
\vdots \\
0 \\
\bar{T}_{N}(t)
\end{array}\right) \text {. This latter ends the proof with regard to (1) where } \\
& y=g(x)^{T} \frac{\partial H(x)}{\partial x} .
\end{aligned}
$$

Remark 1. It can be shown that the dissipation term using (3) with $R(x)$ (defined in (14)) is negative. Indeed, we have:

$$
d=-x^{T} R(x) x=-\frac{D}{h^{2}}\left(\sum_{i=1}^{N-2}\left(x_{i}-x_{i+1}\right)^{2}+x_{1}^{2}+x_{(N-1)}^{2}\right)<0 .
$$

Equality in (15) holds only if $x_{1}=x_{2}=\ldots=x_{N-1}=0$. Consequently, the passivity inequality (4) holds in strict sense (i.e., $\left.\frac{d H(x)}{d t}<u^{T} y\right)$.

\subsection{Feedback controller synthesis}

In what follows, a state feedback control law is proposed to stabilize the PH system of Proposition 1 where all the state variables are assumed to be available online. This result is stated in Proposition 2 .

Proposition 2. Feedback controller synthesis

Under the available online measurement assumption ${ }^{4}$, a (simple) proportional static output feedback law given by,

$$
u=-K y
$$

\footnotetext{
${ }^{4}$ From a mathematical point of view, this consists in considering the so-called observability matrix. A weaker requirement that can also be considered is the detectability condition. We shall not elaborate any further on these concepts here and refer the reader to $[28,31]$ for more information.
} 
where the gain matrix $K \in \Re^{(N-1) \times(N-1)}$ is symmetric and positive definite (i.e., $K=K^{T}>$ $0)$, asymptotically stabilizes the PH system of Proposition 1.

\section{Proof.}

The proof follows immediately using (2)(3) and (15) with the feedback law given by (16). Indeed, we have:

$$
\frac{d H(x)}{d t}<-y^{T} K y<0
$$

for $y \neq 0$ since $K=K^{T}>0$. The Hamiltonian storage function given by $H(x)=\frac{1}{2} x^{T} x$ is bounded from below by 0 and its time derivative given by (17) is negative. Thanks to LaSalle's invariance principle [13], the (largest) invariant set associated to $\frac{d H(x)}{d t}=0$ reduces to the origin only so $H(x)$ plays role of Lyapunov function for the asymptotic stabilization of the $\mathrm{PH}$ system dynamics of Proposition 1 at the origin. As a consequence, $x=x(t) \underset{t \rightarrow+\infty}{\longrightarrow} 0$ and therefore, $T_{i}(t) \underset{t \rightarrow+\infty}{\longrightarrow} T_{i}^{*}, i=1 \ldots(N-1)$.

Remark 2. The result given in Proposition 2 is still valid when $N \rightarrow+\infty$ as soon as Eq. (15) holds.

Remark 3. The explicit expressions for the manipulated variables $T_{l}(t)$ and $T_{r}(t)$ to stabilize the temperature $T(z, t)(6)$ at its stationary profile $T^{*}(z)$ can be derived from (16):

$$
\left(\begin{array}{c}
T_{l}(t)-T^{*}(0) \\
0 \\
\vdots \\
T_{r}(t)-T^{*}(L)
\end{array}\right)=-K \frac{D}{h^{2}}\left(\begin{array}{c}
T_{1}(t)-T_{1}^{*} \\
T_{2}(t)-T_{2}^{*} \\
\vdots \\
T_{(N-1)}(t)-T_{(N-1)}^{*}
\end{array}\right), \quad K \in \Re^{(N-1) \times(N-1)}, K=K^{T}>0 .
$$

Or (18) is equivalent to:

$$
\left(\begin{array}{c}
T_{l}(t) \\
0 \\
\vdots \\
T_{r}(t)
\end{array}\right)=-K \frac{D}{h^{2}}\left(\begin{array}{c}
T_{1}(t)-T_{1}^{*} \\
T_{2}(t)-T_{2}^{*} \\
\vdots \\
T_{(N-1)}(t)-T_{(N-1)}^{*}
\end{array}\right)+\left(\begin{array}{l}
T^{*}(0) \\
0 \\
\vdots \\
T^{*}(L)
\end{array}\right), \quad K \in \Re^{(N-1) \times(N-1)}, K=K^{T}>0 .
$$

Note also that the result of Proposition 2 works well even if a weaker condition for the gain matrix $K$ (i.e., $K=K^{T} \geq 0$ instead of $K=K^{T}>0$ as used in many instances, see e.g. [14,28,32]) is considered. Indeed, thanks to the feedback law given by (16), it follows from (2)(3)(15) that:

$$
\frac{d H(x)}{d t}=\underbrace{d}_{<0}-\underbrace{y^{T} K y}_{\geq 0} \leq d<0 .
$$

The negative definiteness condition of $\frac{d H(x)}{d t}$ remains true as the previous case (see (17)).

Remark 4. It follows from (17) that the convergence speed of the controlled system dynamics goes faster by increasing the gain matrix $K$ (i.e. the norm of the gain matrix). 
Let us state the following proposition.

Proposition 3. The system dynamics (10) is a purely dissipative (distributed parameter) PH system with the state variable $x=\bar{T}(z, t)$, structure matrices $J(x)=0$ and $R(x)=-D$. Furthermore, the Hamiltonian storage function is non-negative and bounded from below:

$$
H(\bar{T}(z, t))=\frac{1}{2}\left(\frac{\partial \bar{T}(z, t)}{\partial z}\right)^{2}
$$

\section{Proof.}

Eq. (10) in its infinite dimensional nature can be rewritten as follows:

$$
\frac{\partial \bar{T}(z, t)}{\partial t}=D \frac{\partial}{\partial z}\left(\frac{\partial \bar{T}(z, t)}{\partial z}\right)=D \frac{\partial \bar{T}(z, t)}{\partial z} \frac{\partial}{\partial \bar{T}}\left(\frac{\partial \bar{T}(z, t)}{\partial z}\right) .
$$

By comparing Eqs. (1) and (21), the proof immediately follows.

Remark 5. As a consequence of Proposition 3, the system dynamics (10) is asymptotically stabilizable for all passive controllers (for instance, PID controller) $[7,8]$.

\section{SIMULATION}

In this section, numerical simulations to illustrate the theoretical developments are proposed. All numerical values used for the simulations are given in Table 1.

Table 1. Numerical values and physical properties of Copper

\begin{tabular}{|l|l|l|l|ll|ll|l|}
\hline$\lambda(W /(\mathrm{cm} \mathrm{K}))$ & $\rho\left(\mathrm{g} / \mathrm{cm}^{3}\right)$ & $c(\mathrm{~J} /(\mathrm{gK}))$ & $L(\mathrm{~cm})$ & $T_{l}\left({ }^{\circ} \mathrm{C}\right)$ & $T_{r} \quad\left({ }^{\circ} \mathrm{C}\right)$ & $T^{\text {init }}(z) \quad\left({ }^{\circ} \mathrm{C}\right)$ \\
\hline 3.98 & 8.96 & 0.385 & 20 & 150 & 25 & $\frac{T_{r}}{L^{2}} z^{2}-\frac{T_{l}}{L} z+T_{l}$ \\
\hline
\end{tabular}

Let us note that the initial temperature must fulfill the following two physically feasible constraints: $T^{\text {init }}(z=0)=T_{l}$ and $T^{\text {init }}(z=L)=T_{r}$. A simple expression as given in Table 1, $T^{\text {init }}(z)=\frac{T_{r}}{L^{2}} z^{2}-\frac{T_{l}}{L} z+T_{l}$, verifies these conditions.

\subsection{Stationary state analysis}

It is shown that the stationary state of the dynamics (6) subject to (7) is given by:

$$
T^{*}(z)=\frac{\left(T_{r}-T_{l}\right)}{L} z+T_{l} .
$$

Eq. (22) is a decreasing linear function with respect to $z$. Its shape is given in Fig. 2.

\subsection{Closed-loop simulations}

For the closed-loop simulations, let us take $N=10$ and for the sake of simplicity, we choose for the gain matrix (Eq. (19)), $K=\operatorname{diag}(1,0, \ldots, 0,1)^{5}\left(K \in \Re^{(N-1) \times(N-1)}\right)$. It is worth noting that in this control strategy, any feasible choice which guarantees $K=K^{T} \geq 0$ is acceptable, except that,

\footnotetext{
${ }^{5} \operatorname{diag}(\ldots)$ stands for the diagonal matrix.
} 


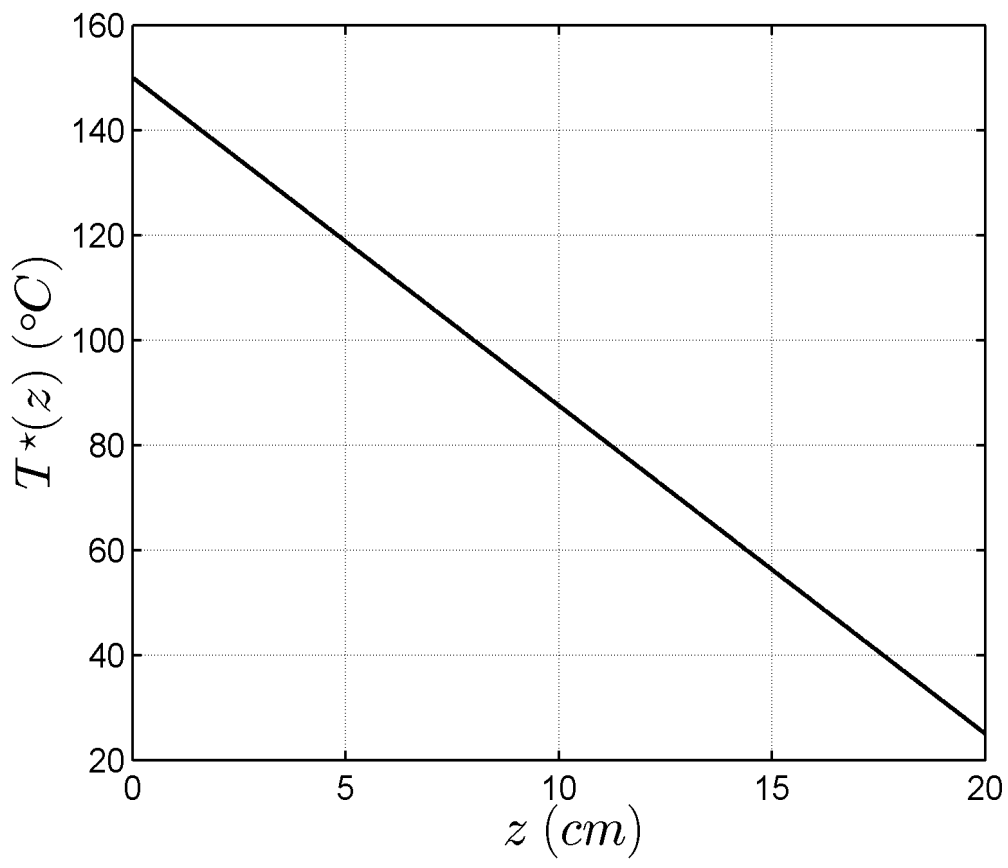

Fig. 2. The distribution of the stationary temperature $T^{*}(z)$

of course, if the gain matrix $K$ is smaller, the controlled system dynamics evolves more slowly (see Remark 4).

Fig. 3 shows that the dynamics of the controlled temperature converges on its stationary value at grid points $z_{i}, \quad i=1 \ldots(N-1)$.

By using the polynomial interpolation of degree $n$ (here $n=5$ is chosen), the profile of the controlled temperature is derived and given in Fig. 4. It is shown that the controlled temperature $T(z, t)$ asymptotically converges on (and coincident with) its stationary value $T^{*}(z)$ at $t \rightarrow+\infty$ (the reader is referred to Fig. 2 for the shape of $T^{*}(z)$ ).

$t=0 t \rightarrow+\infty$

Furthermore, the dynamics of the boundary control inputs $u=\left[\begin{array}{lll}T_{l}(t) & T_{r}(t)\end{array}\right]$ is smooth and physically admissible (see Fig. 5).

Besides, some closed-loop simulations with $N=20$ are given in Fig. 6 and Fig. 7. As shown, the controlled system is stable and has the same behaviours as the previous case (i.e. $N=10$ ).

$$
N=20 N=20
$$

\section{CONCLUSION}

In this work, the control of the temperature profile of one-dimensional unstable heat conduction systems described by parabolic PDEs is presented. Indeed, the authors have shown:

- how to discretize and write the original distributed parameter system dynamics into a $\mathrm{PH}$ representation using the finite difference method. 


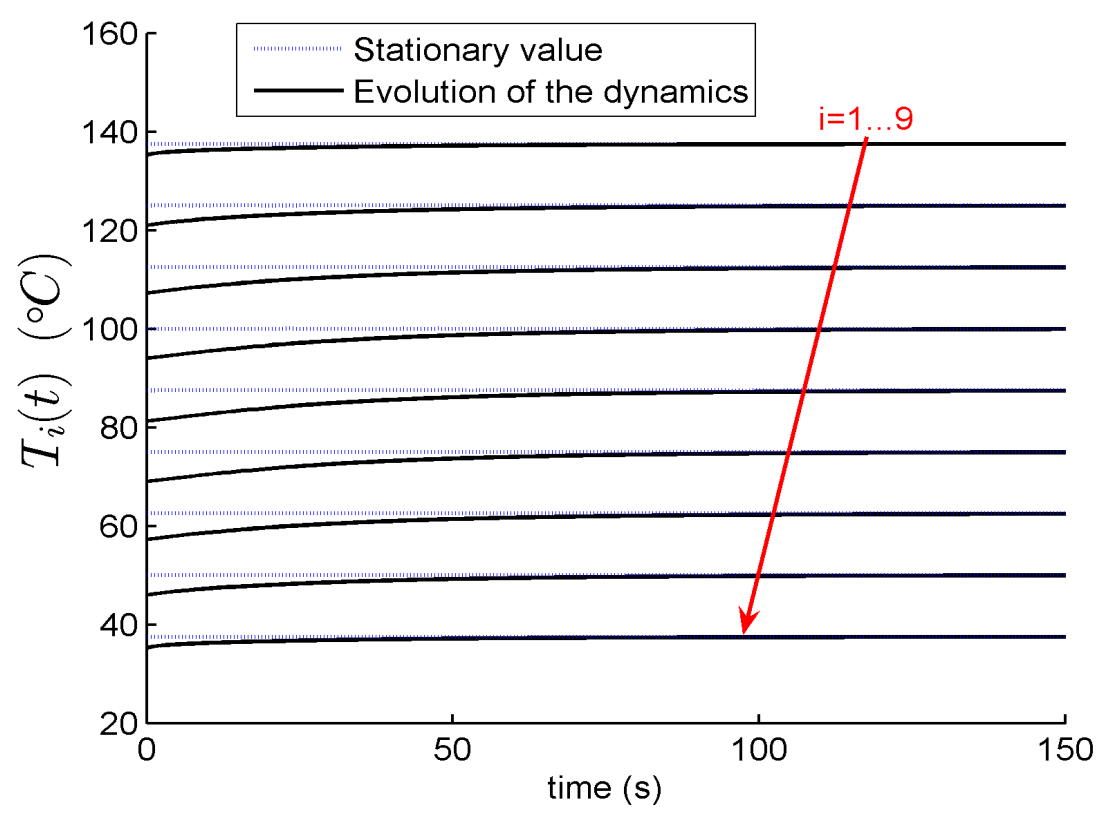

Fig. 3. The controlled temperature

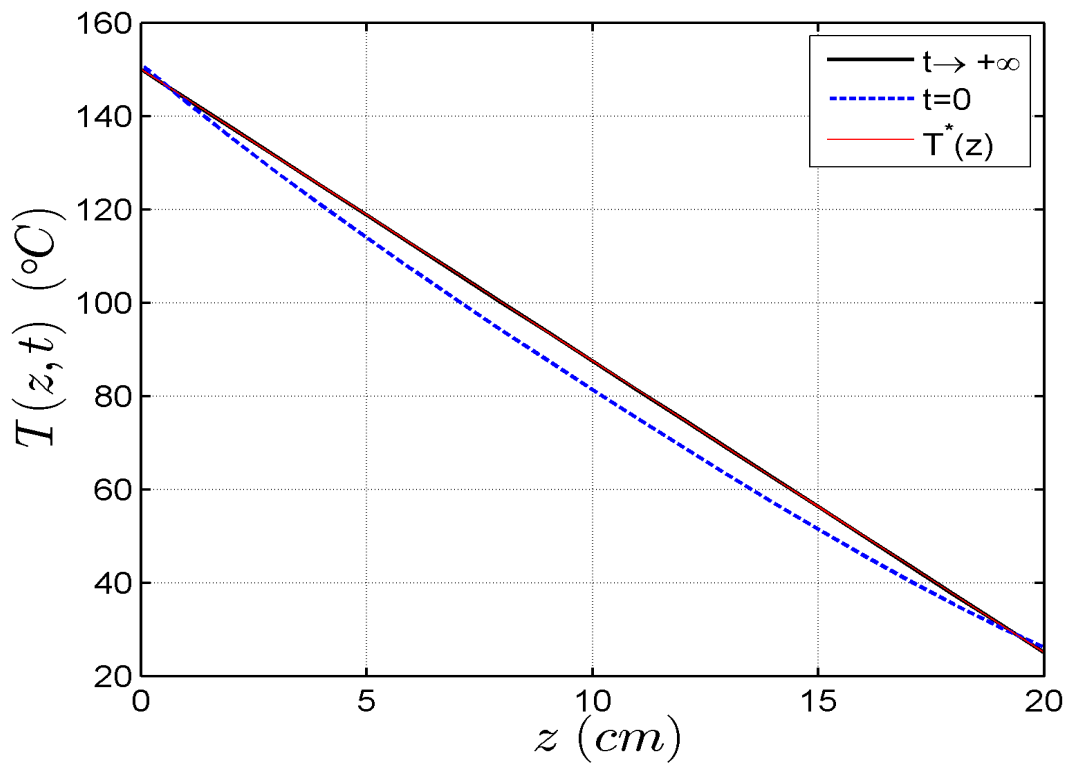

Fig. 4. The profile of the controlled temperature at $t=0$ and $t \rightarrow+\infty$

- how to stabilize that discretized system at a desired reference temperature profile in the PH control framework. Furthermore, the obtained results are also valid even if the step size $h$ is smaller, e.g. $N \rightarrow+\infty$. 

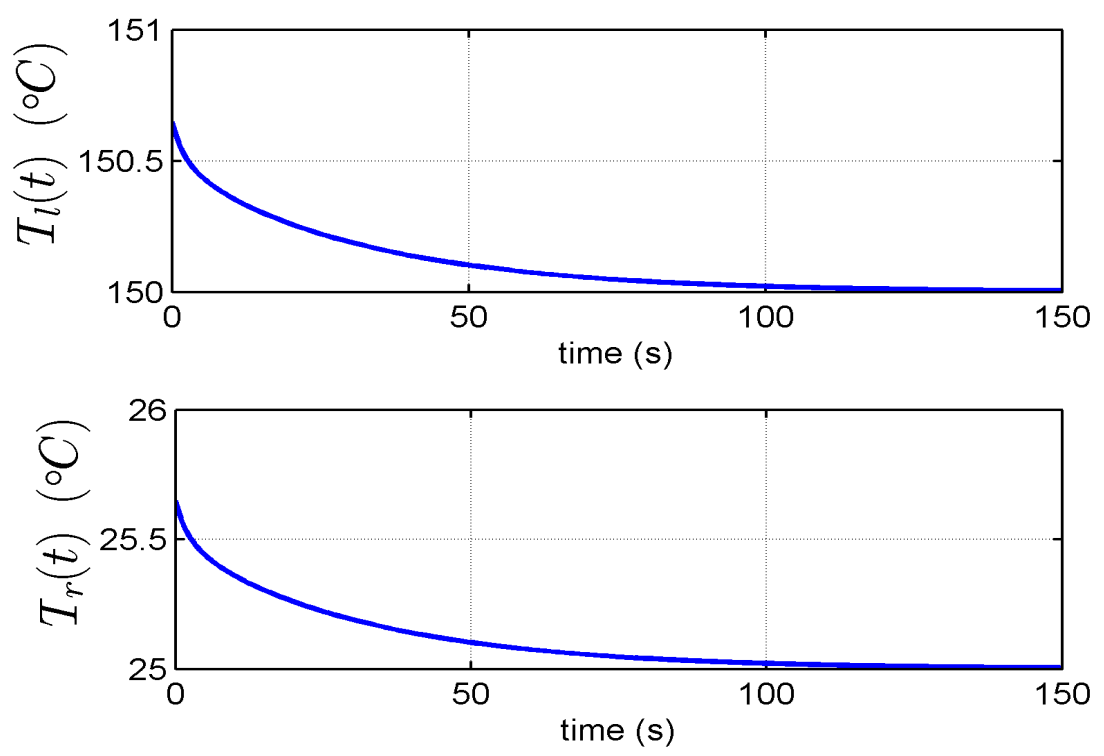

Fig. 5. The boundary control inputs

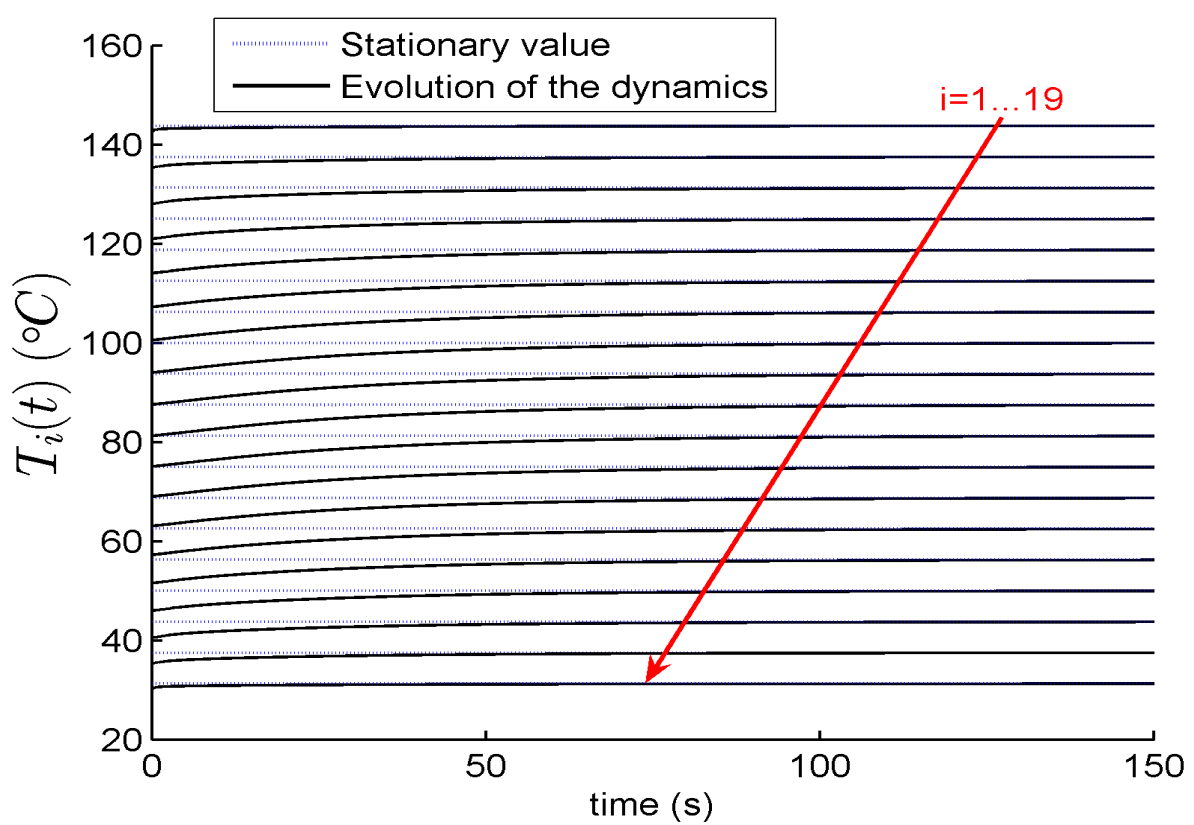

Fig. 6. The closed-loop simulation for the temperature with $N=20$

With regard to infinite dimensional nature, the proposed results are interesting. It is shown that the original distributed parameter system dynamics is an infinite dimensional PH system. In addition, the numerical simulations showed that the convergence objective is satisfied and the boundary control input is physically implementable. It remains now: 

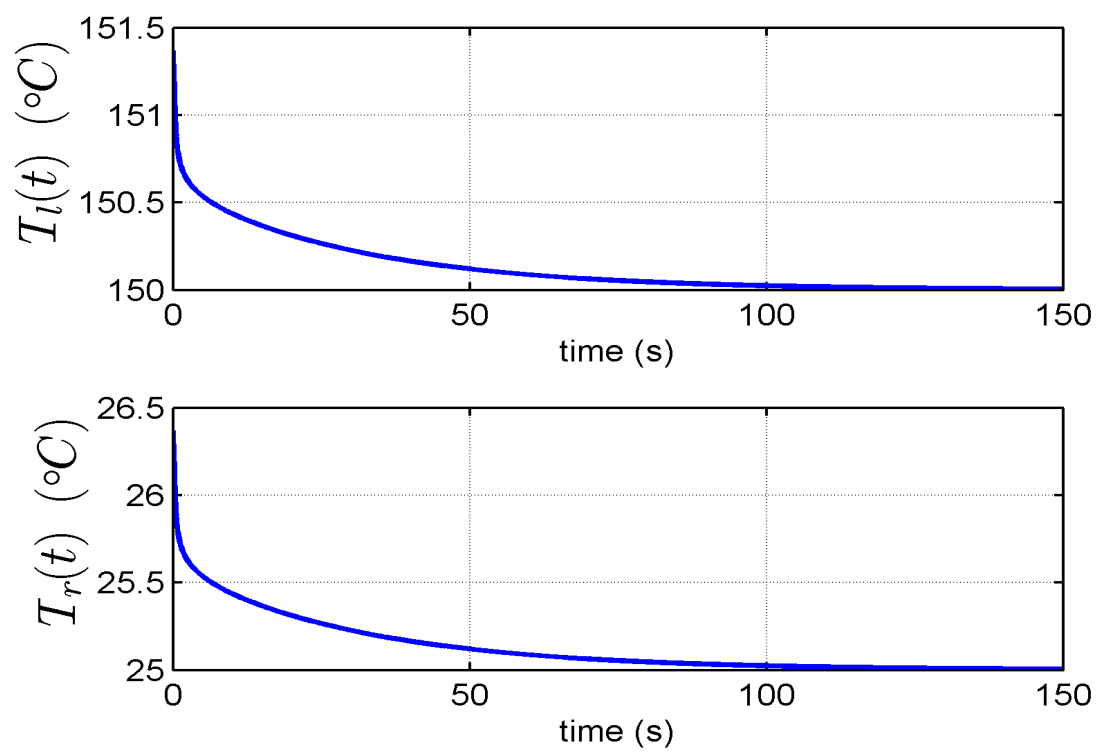

Fig. 7. The closed-loop simulation for the control inputs with $N=20$

- to evaluate and compare the results with the ones developed in $[7,8]$ (on the basis of thermodynamics and the passivity theory) in terms of the performances and robustness.

- to couple the proposed controller with an observer since all the state variables are used.

- to extend the proposed method to more complex distributed parameter systems with chemical reaction (see e.g., $[25,33])$.

\section{ACKNOWLEDGMENTS}

This research is funded by Viet Nam National Foundation for Science and Technology Development (NAFOSTED) under grant number 104.99-2014.74.

\section{REFERENCES}

[1] S. d. Groot and P. Mazur, "Non-equilibrium thermodynamics," 1962.

[2] S. Sandler, "Chemical and engineering thermodynamics," 1999.

[3] W. Luyben, "Process modeling, simulation, and control for chemical engineers," 1990.

[4] P. D. Christofides, "Control of nonlinear distributed process systems: Recent developments and challenges," AIChE Journal, vol. 47, no. 3, pp. 514-518, 2001.

[5] W. Ray, "Some recent applications of distributed parameter systems theorya survey," Automatica, vol. 14, no. 3, pp. 281-287, 1978.

[6] R. Curtain and H. Zwart, "An introduction to infinite-dimensional linear systems theory," 1995.

[7] M. Ruszkowski, V. Garcia-Osorio, and B. E. Ydstie, "Passivity based control of transport reaction systems," AIChE Journal, vol. 51, no. 12, pp. 3147-3166, 2005. 
[8] A. A. Alonso and B. E. Ydstie, "Stabilization of distributed systems using irreversible thermodynamics," Automatica, vol. 37, no. 11, pp. 1739-1755, 2001.

[9] L. T. Antelo, I. Otero-Muras, J. R. Banga, and A. A. Alonso, "A systematic approach to plantwide control based on thermodynamics," Computers $\& 3$ chemical engineering, vol. 31, no. 5, pp. 677-691, 2007.

[10] R. Burden and J. Faires, "Numerical analysis," 2010.

[11] R. Moulla, L. Lefèvre, and B. Maschke, "Geometric pseudospectral method for spatial integration of dynamical systems," Mathematical and Computer Modelling of Dynamical Systems, vol. 17, no. 1, pp. 85-104, 2011.

[12] H. Peng, A. Baaiu, F. Couenne, and Y. Le Gorrec, "Energy based discretization of a class of distributed parameters processes comparison with classical approach," in Decision and Control, 2007 46th IEEE Conference on. IEEE, 2007, pp. 3513-3518.

[13] R. Burden and J. Faires, "Nonlinear systems," 2002.

[14] A. Van Der Schaft, "L2-gain and passivity techniques in nonlinear control," 2000.

[15] B. Brogliato, R. Lozano, B. Maschke, and O. Egeland, Dissipative systems analysis and control. Springer, 2007.

[16] S. Dubljevic, P. Mhaskar, N. H. El-Farra, and P. D. Christofides, "Predictive control of transportreaction processes," Computers E3 chemical engineering, vol. 29, no. 11, pp. 2335-2345, 2005.

[17] P. D. Christofides, "Robust control of parabolic pde systems," Chemical Engineering Science, vol. 53, no. 16, pp. 2949-2965, 1998.

[18] C. Vilas, M. R. García, J. R. Banga, and A. A. Alonso, "Robust feed-back control of distributed chemical reaction systems," Chemical engineering science, vol. 62, no. 11, pp. 2941-2957, 2007.

[19] N. H. El-Farra, A. Armaou, and P. D. Christofides, "Analysis and control of parabolic pde systems with input constraints," Automatica, vol. 39, no. 4, pp. 715-725, 2003.

[20] B. Hamroun, L. Lefèvre, and E. Mendes, "Passivity based control of a reduced port-controlled hamiltonian model for the shallow water equations," in Decision and Control, 2008. CDC 2008. 47th IEEE Conference on. IEEE, 2008, pp. 3917-3922.

[21] M. Li and P. D. Christofides, "Optimal control of diffusion-convection-reaction processes using reduced-order models," Computers \& chemical engineering, vol. 32, no. 9, pp. 2123-2135, 2008.

[22] C. Qi and H.-X. Li, "A time/space separation-based hammerstein modeling approach for nonlinear distributed parameter processes," Computers \& Chemical Engineering, vol. 33, no. 7, pp. 1247-1260, 2009.

[23] S. Godasi, A. Karakas, and A. Palazoglu, "Control of nonlinear distributed parameter processes using symmetry groups and invariance conditions," Computers 83 chemical engineering, vol. 26, no. 7, pp. 1023-1036, 2002.

[24] H. Ramirez, Y. Le Gorrec, A. Macchelli, and H. Zwart, "Exponential stabilization of boundary controlled port-hamiltonian systems with dynamic feedback," Automatic Control, IEEE Transactions on, vol. 59, no. 10, pp. 2849-2855, 2014.

[25] N. H. Hoang, D. Dochain et al., "A thermodynamic approach to the passive boundary control of tubular reactors," Nonlinear Control Syst, vol. 9, pp. 383-388, 2013. 
[26] A. v. d. SCHAFT, "Robust nonlinear control. port-controlled hamiltonian systems: Towards a theory for control and design of nonlinear physical systems." , vol. 39, no. 2, pp. 91-98, 2000.

[27] B. Maschke, R. Ortega, and A. J. Van Der Schaft, "Energy-based lyapunov functions for forced hamiltonian systems with dissipation," Automatic Control, IEEE Transactions on, vol. 45, no. 8, pp. $1498-1502,2000$.

[28] R. Ortega, A. Van Der Schaft, B. Maschke, and G. Escobar, "Interconnection and damping assignment passivity-based control of port-controlled hamiltonian systems," Automatica, vol. 38, no. 4, pp. 585-596, 2002.

[29] H. Hoang, F. Couenne, C. Jallut, and Y. Le Gorrec, "The port hamiltonian approach to modeling and control of continuous stirred tank reactors," Journal of Process Control, vol. 21, no. 10, pp. $1449-1458,2011$.

[30] M. Zecova, J. Terpak et al., "Usage of the heat conduction model for the experimental determination of thermal diffusivity," in Carpathian Control Conference (ICCC), 201314 th International. IEEE, 2013, pp. 436-441.

[31] F. . Lozano, "Adaptive control systems," 08 Jun 1999.

[32] L. A. N. P. Ortega, R. and H. Sira-Ramrez, "Passivity-based control of euler-lagrange systems: Mechanical, electrical and electromechanical applications," 1998.

[33] T. Nguyen and N. Hoang, "Optimization and simulation in chemical engineering: Application to the production of cyclopentenol from cyclopentadiene," VNU Journal of Science, vol. 30, no. 6S-A, pp. 32-43, 2014.

Received on June 03 - 2015

Revised on April 08 - 2016 